\title{
Lactobacillus salivarius alleviates inflammation via NF-KB signaling in ETEC K88-induced IPEC-J2 cells
}

\author{
Jiayun Qiao ${ }^{1} \mathbb{D}$, Zeyang Sun ${ }^{1}$, Dongmei Liang ${ }^{1}$ and Haihua Li²*
}

\begin{abstract}
Background: Enterotoxigenic Escherichia coli (ETEC) K88 commonly colonize in the small intestine and keep releasing enterotoxins to impair the intestinal barrier function and trigger inflammatory reaction. Although Lactobacillus salivarius (L. salivarius) has been reported to enhance intestinal health, it remains to be seen whether there is a functional role of $L$. salivarius in intestinal inflammatory response in intestinal porcine epithelial cell line (IPEC-J2) when stimulated with ETEC K88. In the present study, IPEC-J2 cells were first treated with L. salivarius followed by the stimulation of ETEC K88 for distinct time period. ETEC K88 adherent status, pattern recognition receptors (PRRs) mRNA, mitogen-activated protein kinase (MAPK) and nuclear factor-kB (NF-kB) activation, the release of pro-inflammation cytokines and cell integrity were examined.

Results: Aside from an inhibited adhesion of ETEC K88 to IPEC-J2 cells, L. salivarius was capable of remarkably attenuating the expression levels of interleukin (IL)-1 $\beta$, tumor necrosis factor-a (TNF-a), IL-8, Toll-like receptor (TLR) 4 , nucleotide-binding oligomerization domain (NOD)-like receptor pyrin domain-containing protein (NLRP) 3 and NLRP6. This alternation was accompanied by a significantly decreased phosphorylation of p38 MAPK and p65 NF-KB during ETEC K88 infection with $L$. salivarius pretreatment. Western blot analysis revealed that $L$. salivarius increased the expression levels of zona occludens 1 (ZO-1) and occludin $(P<0.05)$ in ETEC K88-infected IPEC-J2 cells. Compared with ETEC K88-infected groups, the addition of $L$. salivarius as well as extra inhibitors for MAPKs and NFKB to ETEC K88-infected IPEC-J2 cells had the capability to reduce pro-inflammatory cytokines.

Conclusions: Collectively, our results suggest that L. salivarius might reduce inflammation-related cytokines through attenuating phosphorylation of p38 MAPK and blocking the NF-KB signaling pathways. Besides, L. salivarius displayed a potency in the enhancement of IPEC-J2 cell integrity.
\end{abstract}

Keywords: ETEC K88, Inflammatory response, IPEC-J2, L. salivarius

\footnotetext{
*Correspondence: lihaihuaok@126.com

${ }^{2}$ Tianjin Key Laboratory of Agricultural Animal Breeding and Healthy Husbandry, College of Animal Science and Veterinary Medicine, Tianjin Agricultural University, 22 Jinjing Road, Tianjin 300384, People's Republic of China

Full list of author information is available at the end of the article
}

(c) The Author(s). 2020 Open Access This article is licensed under a Creative Commons Attribution 4.0 International License, which permits use, sharing, adaptation, distribution and reproduction in any medium or format, as long as you give appropriate credit to the original author(s) and the source, provide a link to the Creative Commons licence, and indicate if changes were made. The images or other third party material in this article are included in the article's Creative Commons licence, unless indicated otherwise in a credit line to the material. If material is not included in the article's Creative Commons licence and your intended use is not permitted by statutory regulation or exceeds the permitted use, you will need to obtain permission directly from the copyright holder. To view a copy of this licence, visit http://creativecommons.org/licenses/by/4.0/ The Creative Commons Public Domain Dedication waiver (http://creativecommons.org/publicdomain/zero/1.0/) applies to the data made available in this article, unless otherwise stated in a credit line to the data. 


\section{Background}

ETEC K88, a common environmental pathogen, has been considered as a major cause of diarrhea in postweaned piglets, and then contribute to an increased mortality and poor performance during weaning transition [1]. Since the application of certain antibiotics for food-producing animals has been widely restricted, a growing demand for alternative feed additives and ingredients needs to be satisfied in an attempt to improve health condition, boost productive performance and avoid vexing problems like the development of bacterial resistance. Probiotics are tiny living microorganisms which, when consumed by the host as part of food sources with adequate amounts, could confer numerous benefits on the health condition and serve as a potential strategy for certain disease prevention and treatment. A large number of studies have shown that Lactobacillus, an extensively used species both in humans and animals $[2,3]$, is widely known for its capability to improve intestinal health and promote growth performance. This improvement could be obtained by preventing other pathogenic bacteria from attaching to the intestine [4, 5], alleviating existed intestinal damage [6], restoring impaired intestinal barrier [7] and enhancing inadequate immune system [8]. For instance, with reference to a previous knowledge, L. salivarius B1 was found to facilitate an early colonization in duodenal mucosa and an up-regulated expression of porcine $\beta$-defensin-2 (pBD-2) in saliva might be part responsible for the immune response; this up-regulation was tightly correlated with the administration of successive doses of $L$. salivarius B1 [9]. However, the exact mechanisms concerning $L$. salivarius on health modulation, such as inflammatory response and immunology regulation, have not yet been elucidated.

The intestinal mucosal barrier is an integral part for maintaining the homeostasis of the gut microenvironment. The intestinal epithelial cells are important components of this barrier while the intestinal tight junctions adjacent to individual cells act as another physical structure. This complicated tight junction complex consists of approximately 50 proteins which are basically classified into structural and functional units $[10,11]$. The pivotal role of tight junctions is to seal the gap between epithelial cells and thus restrict microorganisms or other antigens from infiltrating into the systematic circulation [12]. Studies have offered perspectives that the treatment of Lactobacillus could augment the expression levels of various tight junction proteins, including claudin-1, occludin and ZO-1. Since tight junctions are responsible for the proper permeability, these elevated protein levels have presented an efficacy of probiotics in maintaining the regular environment of pig intestinal mucosa and reducing porcine diarrhea incidence $[13,14]$.
TLRs and NOD-like receptors (NLRs), defined as two essential groups of membrane receptors of intestinal epithelial cells, play critical roles in the innate immune system by identifying the conserved pathogen-associated molecular patterns (PAMPs) from various invading microbes [15]. It is well understood that this identification could consequently trigger the activation of MAPK, NF$\mathrm{\kappa B}$ and caspase-1 and after which an assortment of inflammation-related cytokines will be released, such as TNF- $\alpha$, IL- 8 , and IL- $1 \beta$. Thus, these signaling pathways are likely to get involved in the host defensive and inflammatory system [16]. One previous research discovered that $L$. plantarum administration could bring not only the inhibition of TLR4-induced MAPK and NF- $\mathrm{B}$ signaling pathways but also the down-regulation of IL$1 \beta$, TNF- $\alpha$ and IL-6. The above-mentioned alternations of immunostimulatory cytokines offered a possibility that $L$. plantarum could be considered as a key regulator of the innate immune system in the infected individuals and that symptoms of pathogenic ETEC K88-induced diseases might be relieved with the assistance of this strain [17]. Besides, Lactobacillus could also modulate the expression levels of NOD-1 and NOD-2, and the change of which could be likely connected with immunomodulatory activities [18]. However, it remains unclear whether L. salivarius could hinder the ETEC K88 infection by mediating cellular immunity via regulating TLRs, NLRs and corresponding downstream targets.

Even though a number of investigators have clarified promising outcomes on the health regulation brought by several Lactobacillus strains, the knowledge about the potential influence of $L$. salivarius on the inflammatory response is still ambiguous. In this study, ETEC K88 was selected as a pathogen to infect IPEC-J2 cells and then a variety of parameters were measured with the treatment of $L$. salivarius, including the expression levels of certain molecules involved in the pathogenic recognition, activation of MAPK or NF- $\mathrm{kB}$ signaling pathways, the secreted cytokines as well as the cell integrity. Our study aims to explore the protective role of $L$. salivarius in regulating inflammatory response when pathogenic invasion occurs in the intestinal epithelial cells.

\section{Materials and methods}

\section{The culture of IPEC-J2 cells}

IPEC-J2 cells were cultured in 89\% Roswell Park Memorial Institute (RPMI) Medium 1640 basic (Gibco, Grand Island, NY, USA) supplemented with 10\% fetal bovine serum (Gibco, Grand Island, NY, USA) and $1 \%$ penicillin/streptomycin (Solarbio, Beijing, China) at $37^{\circ} \mathrm{C}$ with $5 \% \quad \mathrm{CO}_{2}$ in the humidified incubator. After growing to $80-90 \%$ confluence, the cultured cells were digested with $0.25 \%$ trypsin-EDTA for later passage or protein extractions. 


\section{Bacterial strains}

L. salivarius was isolated and purified from fresh feces of 28-day-old healthy weaned-piglets. Certain biochemical methods for identifying those isolated L. salivarius samples were used, such as the gene sequencing of $16 \mathrm{~S}$ rRNA and the homology analysis. L. salivarius was first incubated in Lactobacillus MRS broth at $37^{\circ} \mathrm{C}$ for $24 \mathrm{~h}$. After dilution with fresh medium, the cultures were incubated under the same condition until the appearance of logarithmic phase. L. salivarius was harvested from the broth at 5,000 r/min for $10 \mathrm{~min}$ at $4{ }^{\circ} \mathrm{C}$. The precipitated bacteria and the glycerol were mixed with the ratio of 1:2. Meanwhile, the concentration was confirmed through serial dilutions followed by colony forming unit (CFU) counts on de Man, Rogosa and Sharpe (MRS) agar after $24 \mathrm{~h}$ incubation under anaerobic environment. All the cultured bacteria were finally stored at $-80{ }^{\circ} \mathrm{C}$ for further performances. The ETEC K88 strains were obtained from the China Veterinary Culture Collection Center (CVCC1502). The performances of bacterial culturing, harvesting, and counting were conducted as previously described [8].

\section{ETEC K88 adhesion assay}

IPEC-J2 cells were inoculated into two 24-well plates (Corning, Inc., Corning City, NY, USA) with a density of $3 \times 10^{5}$ cells/well for $50 \%$ confluence. L. salivarius $(1 \times$ $10^{5} \mathrm{CFU} /$ well) were then supplemented into these wells for $3 \mathrm{~h}$, and after which cells were stimulated with ETEC K88 $\left(1 \times 10^{3} \mathrm{CFU} /\right.$ well $)$ for $0,3,6$ and $24 \mathrm{~h}$, respectively. The applied concentration and stimulation time of both L. salivarius and ETEC K88 were designed based on a standard that the normal adherent status of bacteria was not affected while cell monolayer was not destroyed. Pig lactate dehydrogenase (LDH) enzyme-linked immunosorbent assay (ELISA) Kit was utilized to detect whether the cellular structure was damaged. IPEC-J2 cells with different treatments were washed three times with RPMI Medium 1640 basic to remove non-adherent ETEC K88. $100 \mu \mathrm{L}$ of $0.5 \%$ TritonX-100 was then added to each well of the plate to lyse the cells for $8 \mathrm{~min}$ in the $37^{\circ} \mathrm{C}$ incubator, and $900 \mu \mathrm{L}$ of phosphate buffer saline (PBS) was used to stop the cell lysis. The lysate was serially diluted and incubated in Luria-Bertani (LB)-Agar dishes for 12 $\mathrm{h}$ to quantitate bacterial populations. At this time, the adherent population of ETEC K88 (C1) was the total adherent population of both phagocytes and those ETEC K88 sticking to the surface of IPEC-J2 cells. The other plate was added with $0.4 \mathrm{~mL}$ culture medium containing $100 \mu \mathrm{g} / \mathrm{mL}$ gentamycin per well for $2 \mathrm{~h}$ to kill IPEC-J2 cells with ETEC K88 attachment on the cellular surface. The gentamicin was finally discarded and the cells were lysed and terminated as described above. In this case, the remained cells in fact were those with engulfed
ETEC K88 and the phagocytosis population of ETEC K88 (C2) was correspondingly calculated. ETEC K88 adherent population $=\mathrm{C} 1-\mathrm{C} 2$. The adherent ratio $=(\mathrm{C} 1$ $-\mathrm{C} 2) / \mathrm{C} 1 \times 100 \%$.

\section{Quantitative real-time polymerase chain reaction (qPCR)}

IPEC-J2 cells were pretreated with $L$. salivarius and followed by ETEC K88 infection as described above. All the treated IPEC-J2 cells were harvested at 3, 6 or $12 \mathrm{~h}$ post-infection, respectively, for total RNA extraction by using TRIzol reagent (Takara Biotechnology, Dalian, China). One $\mu \mathrm{g}$ of qualified RNA was used for cDNA synthesis using Moloney Murine Leukemia Virus Reverse Transcriptase (Promega, Madison, WI, USA) to later examine the expression levels of TLR2, TLR4, NLRP3 and NLRP6. The qPCR was performed based on designed primers (Table 1) and the relative expressions of target genes were analyzed as previously described [8]. All samples were run in triplicate.

\section{Cellular lysate preparation}

IPEC-J2 cells were lysed with protease inhibitor-lysis solution for $10 \mathrm{~min}$ on ice, and cell debris were then removed by centrifugation at 12,000 r/min for $15 \mathrm{~min}$ at $4{ }^{\circ} \mathrm{C}$. Total protein concentration was determined using the BCA Protein Assay Kit (Applygen Technologies Inc., Beijing, China).

\section{Western blotting}

The extracted proteins from different treated cells were mixed with Laemmli sample buffer for 12\% SDS-PAGE and a consistent amount of each protein sample was loaded. After electrophoresis, the gel with separated proteins was released from the plastic case and those protein samples were electrophoretically transferred onto polyvinylidene difluoride (PVDF) membrane (Millipore, Billerica, MA, USA). This membrane with migrated proteins was then coated with blocking buffer for $2 \mathrm{~h}$ at

\begin{tabular}{lllc}
\multicolumn{4}{c}{ Table $\mathbf{1}$ Sequences of oligonucleotide primers used for qPCR } \\
\hline Target & Primer & Sequence (5' to 3') & Size, bp \\
\hline TLR2 & Forward & TCATCTCCCAAATCTGCGAT & 167 \\
& Reverse & GGCTGATGTCTGAATTGACCTC & \\
TLR4 & Forward & CCGTCATTAGTGCGTCAGTTCT & 100 \\
& Reverse & TTGCAGCCCACAAAAAGCA & \\
NLRP3 & Forward & AGCAGATTCCAGTGCATCAAAG & 76 \\
& Reverse & CCTGGTGAAGCGTTGTGAG & \\
NLRP6 & Forward & TCAACCGCCTCTTCAGCC & 116 \\
& Reverse & CGCCCAGTCGTACAGGATTT & \\
GAPDH & Forward & GAAGGTCGGAGTGAACGGAT & 150 \\
& Reverse & CATGGGTAGAATCATACTGGACA & \\
\hline
\end{tabular}


room temperature to prevent unspecific bindings and incubated with optimized diluted primary anti-bodies for proteins of interests for $2 \mathrm{~h}$ at room temperature, including anti-ZO-1 (Abcam, Cambridge, UK), antioccludin (Abcam, Cambridge, UK), anti-NF-кB p65 (Cell Signaling Technology, Danvers, MA, USA), antiphosphо-NF-кB p65 (Cell Signaling Technology, Danvers, MA, USA), anti-p38 MAPK (Cell Signaling Technology, Danvers, MA, USA), anti-phospho-p38 MAPK (Cell Signaling Technology, Danvers, MA, USA), antiERK MAPK (Cell Signaling Technology, Danvers, MA, USA), anti-phospho-ERK MAPK (Cell Signaling Technology, Danvers, MA, USA) and anti-GAPDH (Abcam, Cambridge, UK) antibodies. After 3 times rinsing with Tris-Buffered-Saline with Tween (TBST), the membrane was incubated with horseradish peroxidase (HRP)-conjugated secondary antibodies for $1 \mathrm{~h}$ at room temperature. The immunoblots were carried out for imaging with the Western blot luminescence detection kit (Santa Cruz Biotechnology, Santa Cruz, CA, USA), and the presences of ZO-1, occludin, NF-кB p65, p38 MAPK, ERK MAPK, and GAPDH were automatically exposed by AlphaImager 2200 (Alpha Innotech, San Leandro, CA, USA) with an appropriate exposure time. Band densities were finally quantified using AlphaImager 2200 as well (Alpha Innotech, San Leandro, CA, USA).

\section{LDH assay}

LDH, a cytoplastic enzyme, will be released into the bloodstream when there is a damage to cells. Therefore, after previously described treatments, the supernatants containing released LDH of cultured IPEC-J2 cells were collected to determine the integrity of cells. IPEC-J2 cells were first harvested and resuspended with $\mathrm{LDH}$ assay buffer. After centrifugation at 12,000 r/min for $5 \mathrm{~min}$ at $4{ }^{\circ} \mathrm{C}$, supernatants were carefully collected and transferred into the clean tubes. Reaction mix for the assay was prepared by using LDH assay kit (Nanjing Jiancheng Bioengineering Institute, Nanjing, China) according to the manufacturer's instructions and added into each well with the sample or the control. The fluorescence was immediately measured at $450 \mathrm{~nm}$ and the obtained values were analyzed based on the protocol to monitor the activity of LDH. Each sample was assayed for three replicates.

\section{Analysis of cytokines}

IPEC-J2 cells were seeded into 24-well culture plates and used for later experiments until the cell density reached $80 \%$ confluence. IL- $1 \beta$, TNF- $\alpha$ and IL- 8 production were measured from the supernatants of IPEC-J2 cells treated or untreated with ETEC K88 $\left(1 \times 10^{3} \mathrm{CFU} /\right.$ $\mathrm{mL})$ or L. salivarius $\left(1 \times 10^{5} \mathrm{CFU} / \mathrm{mL}\right)$ either alone or simultaneously coupled with ETEC K88 for $3 \mathrm{~h}$ till $24 \mathrm{~h}$.
The cells only treated with $L$. salivarius were set as the control group. Distinct cytokine levels were quantitatively assessed by ELISA using a commercial ELISA kit (Nanjing Jiancheng Biology Engineering Institute, Nanjing, China).

\section{The functions of MAPK and NF-KB involved in TLRs- and NLRs-triggered signaling pathways during the process of Lactobacilli regulating the inflammatory response}

SB-203580 (MAPK inhibitor, GlpBio, Montclair, CA, USA), SCH772984 (ERK inhibitor, GlpBio, Montclair, CA, USA) and BAY11-7082 (NF- $\mathrm{kB}$ inhibitor, GlpBio, Montclair, CA, USA) were first employed in IPEC-J2 cells for $1 \mathrm{~h}$ [19-21], followed by $L$. salivarius involvement for $3 \mathrm{~h}$. After 3 times washes with PBS, IPEC-J2 cells were infected with ETEC K88 for 3, 6, 12 and 24 h, respectively. Non-infected cells were considered as the control group. The cell supernatants were collected from each group, and then the pro-inflammatory cytokines, including IL- $1 \beta$, TNF- $\alpha$, IL- 8 , were detected by ELISA according to the manufacture's protocols.

\section{Statistical analysis}

All experiments were performed with at least three independent replicates. Statistical analysis was completed using GraphPad Prism 5, and differences were evaluated by Student's $t$-test. $P$-value of $<0.05$ was considered statistically significant.

\section{Results}

Inhibited adhesion of ETEC K88 to IPEC-J2 cells

The adhesion of ETEC K88 to L. salivarius-pretreated IPEC-J2 cells was reduced to varying degrees during the infection. The ratio of the adherent rate of ETEC in cells added with $L$. salivarius to that in the control group was 64\% $(P=0.0081), 67 \%(P=0.0091), 80 \%(P=0.1236)$, and $94 \%(P=0.3739)$, respectively (Fig.1). Internalization of ETEC K88 by IPEC-J2 was not observed.

\section{Effects of $L$. salivarius on TLRs- and NLRs-mediated signaling pathways}

To investigate the effects of $L$. salivarius on the inflammatory process triggered in ETEC K88-challenge IPECJ2 cells, mRNA levels of TLRs and NLRs were basically detected in three different groups, namely the group of IPEC-J2 cells treated with ETEC K88 alone, the group of cells preincubated with $L$. salivarius before ETEC K88 infection and the control group without any stimulation. After ETEC K88 stimulation for 3, 6 and $12 \mathrm{~h}$, the mRNA level of TLR2 was significantly higher in IPEC-J2 cells with $L$. salivarius supplementation when compared with either the control group or ETEC K88 alone group. $(P<0.01$, Fig. 2a). However, there was no obvious difference of TLR2 mRNA observed between ETEC K88 alone 


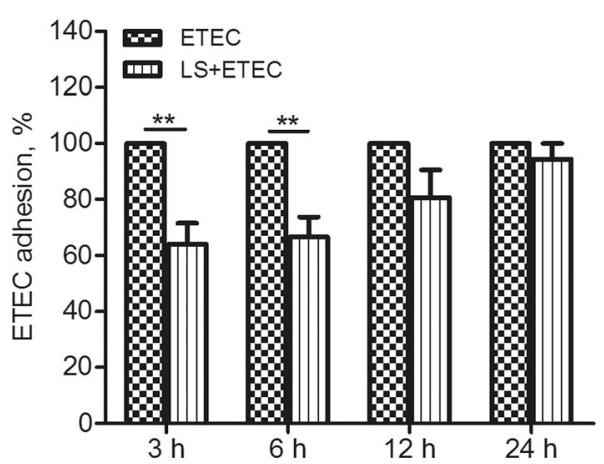

Fig. 1 Effects of L. salivarius on ETEC K88 adhesion to IPEC-J2. Cells were collected from the indicated IPEC-J2 cultures at 3, 6, 12, $24 \mathrm{~h}$ after ETEC K88 challenge. An adhesion assay using ETEC K88 alone served as a reference, and the adherent ratio of ETEC K88 group was normalized to $100 \%$. Data are presented as means \pm standard deviations (SD) of three independent experiments. ${ }^{* *}, P<0.01$

group and $L$. salivarius pretreated one with a constant challenge from ETEC K88 for $24 \mathrm{~h}(P>0.05)$. The qPCR analysis also showed a pronounced down-regulation of TLR4 mRNA in L. salivarius pretreated cells compared with ETEC K88 alone group at 3, 6, 12 and $24 \mathrm{~h}$ after ETEC K88 challenge $(P<0.05$ or $P<0.01$, Fig. $2 \mathrm{~b})$.
Besides, there was a significant elevation of mRNA levels for both NLRP3 and NLRP6 in cells preincubated with L. salivarius and ETEC K88 infectious time for them was $3 \mathrm{~h}$ or $6 \mathrm{~h}$ (Fig. $2 \mathrm{c}$, d), but the expression level of NLRP3 started to decline after $6 \mathrm{~h}$ infection while that of NLRP6 began to drop after $12 \mathrm{~h}$ infection (Fig. $2 \mathrm{c}, \mathrm{d}$ ).

\section{Activation of MAPK and NF-KB signaling pathways in ETEC K88-stimulated IPEC-J2 cells}

To investigate the potential signaling pathways that were responsible for the release of inflammation-related cytokines, the phosphorylation of certain proteins, specifically the MAPK family and NF- $\mathrm{KB}$ were examined by Western blotting (Fig. 3). The result indicated that p38 MAPK, ERK MAPK, as well as p65 NF-kB were rapidly phosphorylated with ETEC K88 stimulation in IPEC-J2 cells. Interestingly, the phosphorylation of ERK was markedly increased in L. salivarius-pretreated IPEC-J2 cells compared with ETEC K88 alone group after ETEC K88 infection for $1 \mathrm{~h}(P<0.01)$ or $3 \mathrm{~h}(P<0.05)$ (Fig. 3a). However, L. salivarius enabled phosphorylation of p38 and p65 to substantially decline in cells infected with ETEC K88 for either $3 \mathrm{~h}$ or $6 \mathrm{~h}$ when compared with ETEC K88 alone group $(P<0.05$ or $P<0.01$; Fig. $3 \mathrm{~b}, \mathrm{c})$.
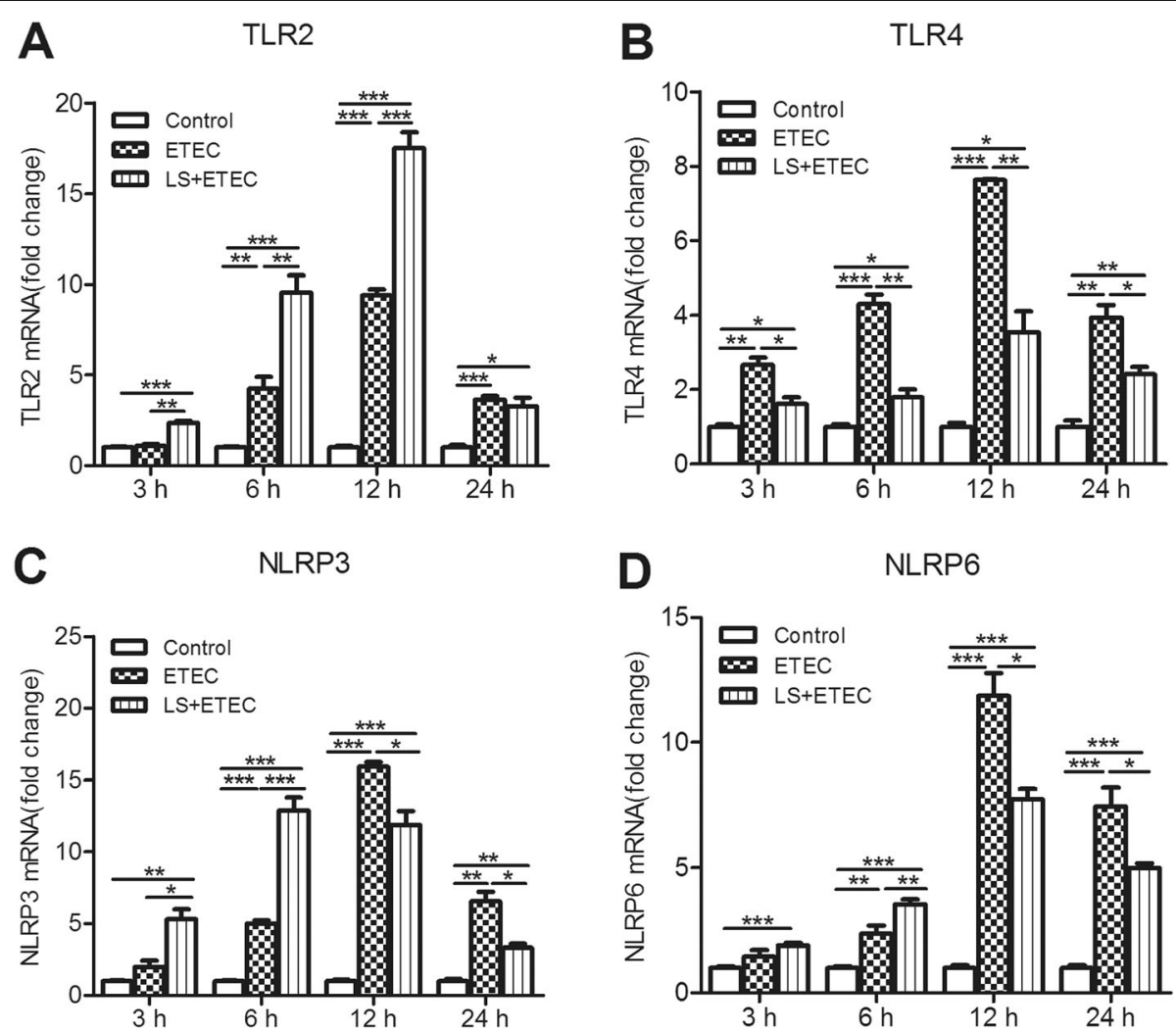

D

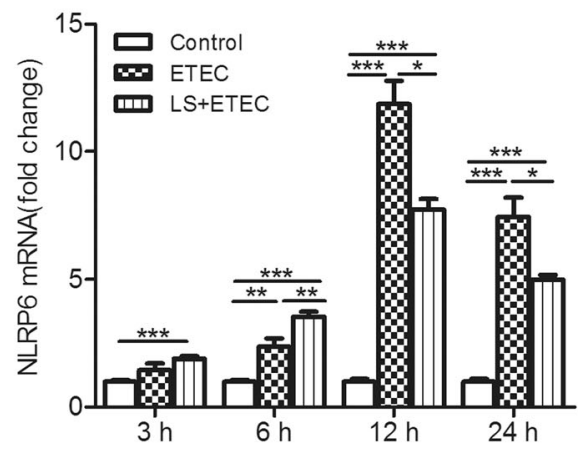

Fig. 2 Effects of L. salivarius on TLR- and NOD-mediated inflammatory signaling pathways. IPEC-J2 cells collected from the 6-well dishes at 3, 6, 12, $24 \mathrm{~h}$ after ETEC K88 challenge. The relative expressions of mRNA for (a) TLR2, (b) TLR4, (c) NLRP3 and (d) NLRP6 were analyzed by quantitative real-time PCR. Data are presented as means \pm SEM of three independent experiments. ${ }^{*}, P<0.05 ;{ }^{* *}, P<0.01$; ${ }^{* *}, P<0.001$ 
The cytokine alterations with Lactobacillus pretreatment Inflammatory status of IPEC-J2 cells was evaluated by measuring the expression levels of pro-inflammatory cytokines (IL-1 $\beta$, IL- 8 and TNF- $\alpha$ ). ETEC K88 treatment for $6 \mathrm{~h}$ till $24 \mathrm{~h}$ resulted in the increased levels of IL-1 $\beta$ and IL- $8(P<0.05$ or $P<0.01$, Fig. $4 \mathrm{a}-\mathrm{b})$ whereas $3 \mathrm{~h}$ till $24 \mathrm{~h}$ infection induced a significant augmentation of TNF- $\alpha$ (Fig. 4 c). Meanwhile, the assistance of L. salivarius obviously decreased the expression levels of the above three induced cytokines (Fig. 4 a-c). It is worth noticing that there were no significant distinctions of IL$1 \beta$, IL-8, and TNF- $\alpha$ levels between the L. salivarius-pretreated cells and the control ones when ETEC K88 challenging time was $3 \mathrm{~h} .(P>0.05)$.

\section{Positive influence of $L$. salivarius on expression levels of tight junction proteins}

To investigate the possible membrane barrier disruption caused by ETEC K88 and the potential role of L. salivarius in restoring such damage, an immunobolt of $\mathrm{ZO}-1$
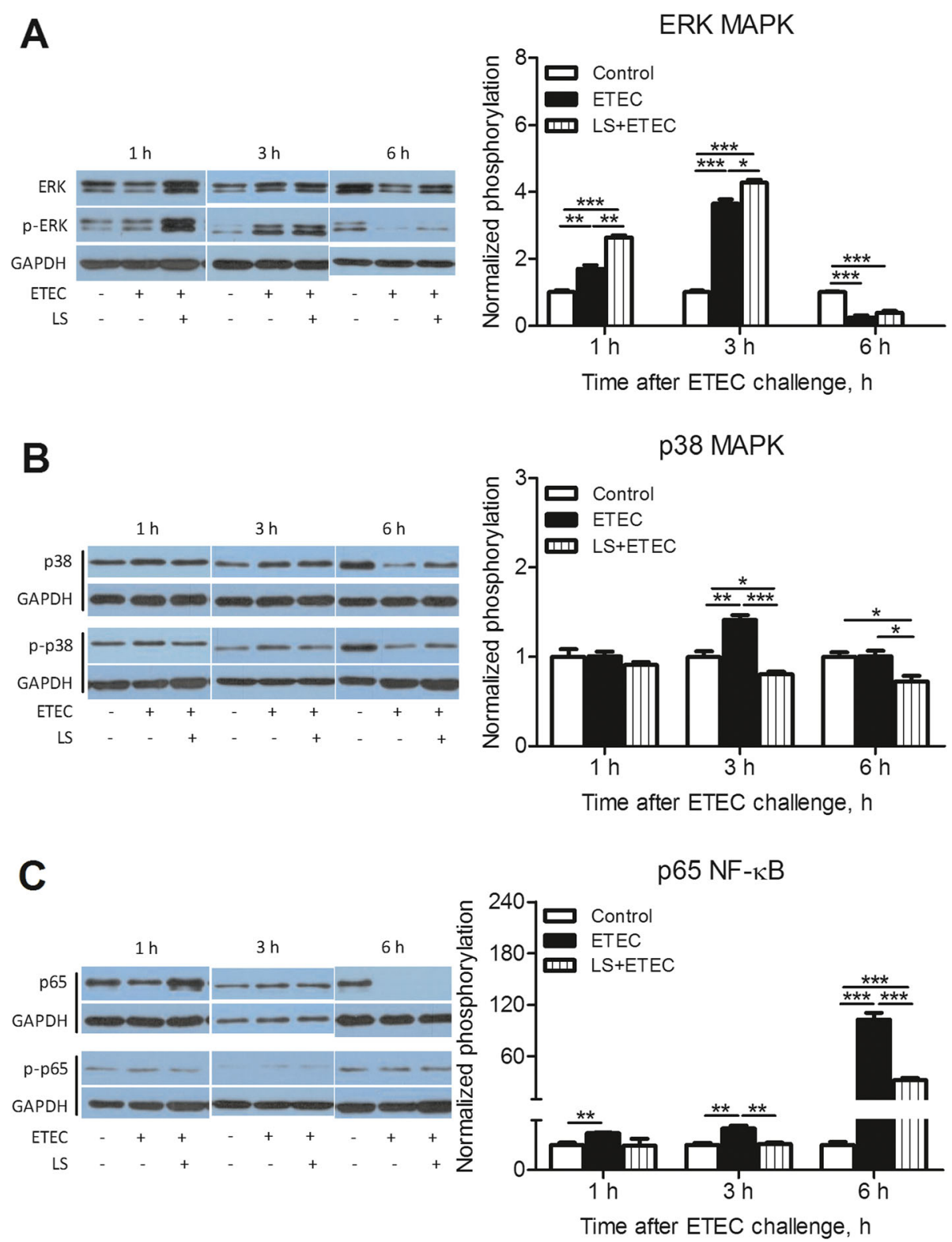

Fig. 3 Activation of MAPK and NF-KB pathways in ETEC K88-stimulated IPEC-J2 cells. IPEC-J2 cells harvested from L. salivarius involvement groups and control ones were treated with ETEC K88, and cell lysates were subjected to Western blotting with phosphor-specific Abs against (a) p-ERK, (b) p-p38 and (c) p-p65. GAPDH was used as the loading control. Data are presented as means \pm SEM of three independent experiments. ${ }^{*}, P<$ $0.05 ; * *, P<0.01 ; * * *, P<0.001$ 
and occludin was performed (Fig. 5). ETEC K88 was able to obviously decrease expression levels of ZO-1 and occludin in IPEC-J2 cells $(P<0.05)$ while $L$. salivarius involvement prior to infection had the capability to significantly increase the levels of these two tight junction proteins $(P<0.05$, Fig. 5$)$.

\section{Effects of $L$. salivarius on LDH production}

To identify whether ETEC K88 caused cell membrane damage, LDH in IPEC-J2 cell culture medium was detected. Challenge with ETEC K88 for 12 and $24 \mathrm{~h}$ significantly increased LDH content in IPEC-J2 cells $(P<$ 0.05 , Fig. 6a), indicating an increased cell permeability. As expected, $L$. salivarius treatment reduced LDH released from the cells (Fig. 6a). In addition, L. salivarius along with either MAPK inhibitors or NF- $\mathrm{kB}$ inhibitors brought an obviously reduced level of LDH concentration in the medium. $(P<0.05$, Fig. $6 \mathrm{~b}-\mathrm{d})$.

The functions of MAPK and NF-KB involved in TLRs- and NLRs-triggered signaling pathways during the process of Lactobacilli regulating the inflammatory response

Since phosphorylation of p38 MAPK was attenuated in ETEC K88-stimulated IPEC-J2 cells in response to $L$. salivarius, the potential effect of specific MAPK inhibitors on levels of IL- $1 \beta$, IL- 8 , and TNF- $\alpha$ production were further examined. IPEC-J2 cells were added with SB203580 (p38 MAPK inhibitor) and SCH772984 (ERK MAPK inhibitor), respectively for $1 \mathrm{~h}$ and then stimulated with ETEC K88 for $24 \mathrm{~h}$. It turned out that the existence of either SB-203580 or SCH772984 could induce the declined levels of IL-1 $\beta$, IL-8, and TNF- $\alpha$ in groups with $L$. salivarius supplementation and the control ones (Fig. 7a, b). BAY11-7082 (NF-kB inhibitor) was also applied for $1 \mathrm{~h}$ before ETEC K88 stimulation for $24 \mathrm{~h}$, aiming to present the potential role of NF- $\mathrm{kB}$ pathway in the process of $L$. salivarius affecting the production of IL- $1 \beta$, IL- 8 , and TNF- $\alpha$. However, the elevating levels of pro-inflammatory cytokines were detected in ETEC K88 infected groups with the supply of L. salivarius (Fig. 7c).

\section{Discussion}

The application of probiotics has been proved to bring a vast array of well-established effects, including the regulation of pro- and anti-inflammatory cytokines, the enhancement of secretory IgA, the production of antibacterial substances, the preservation of intestinal barrier integrity, and the competition with pathogenic

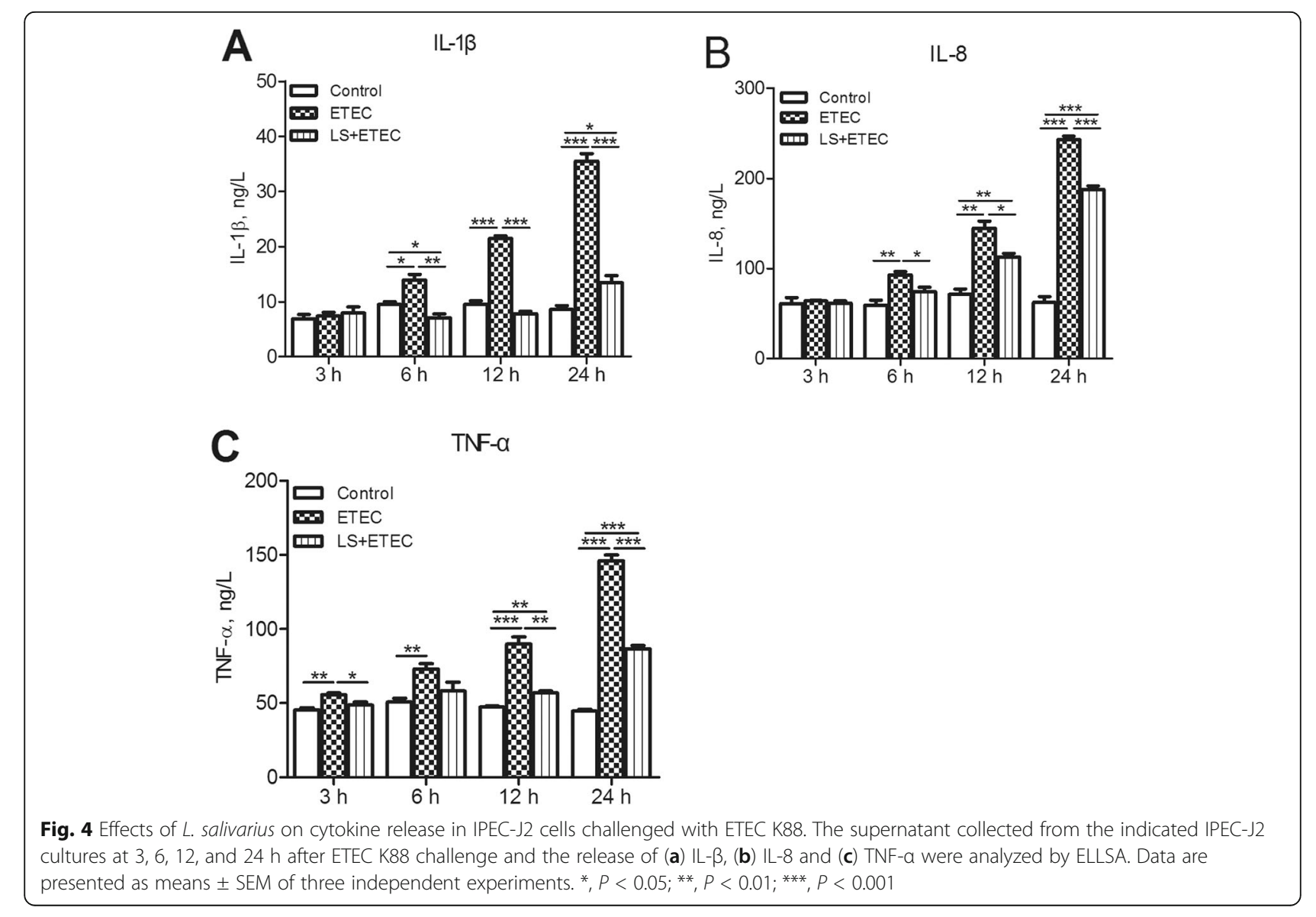



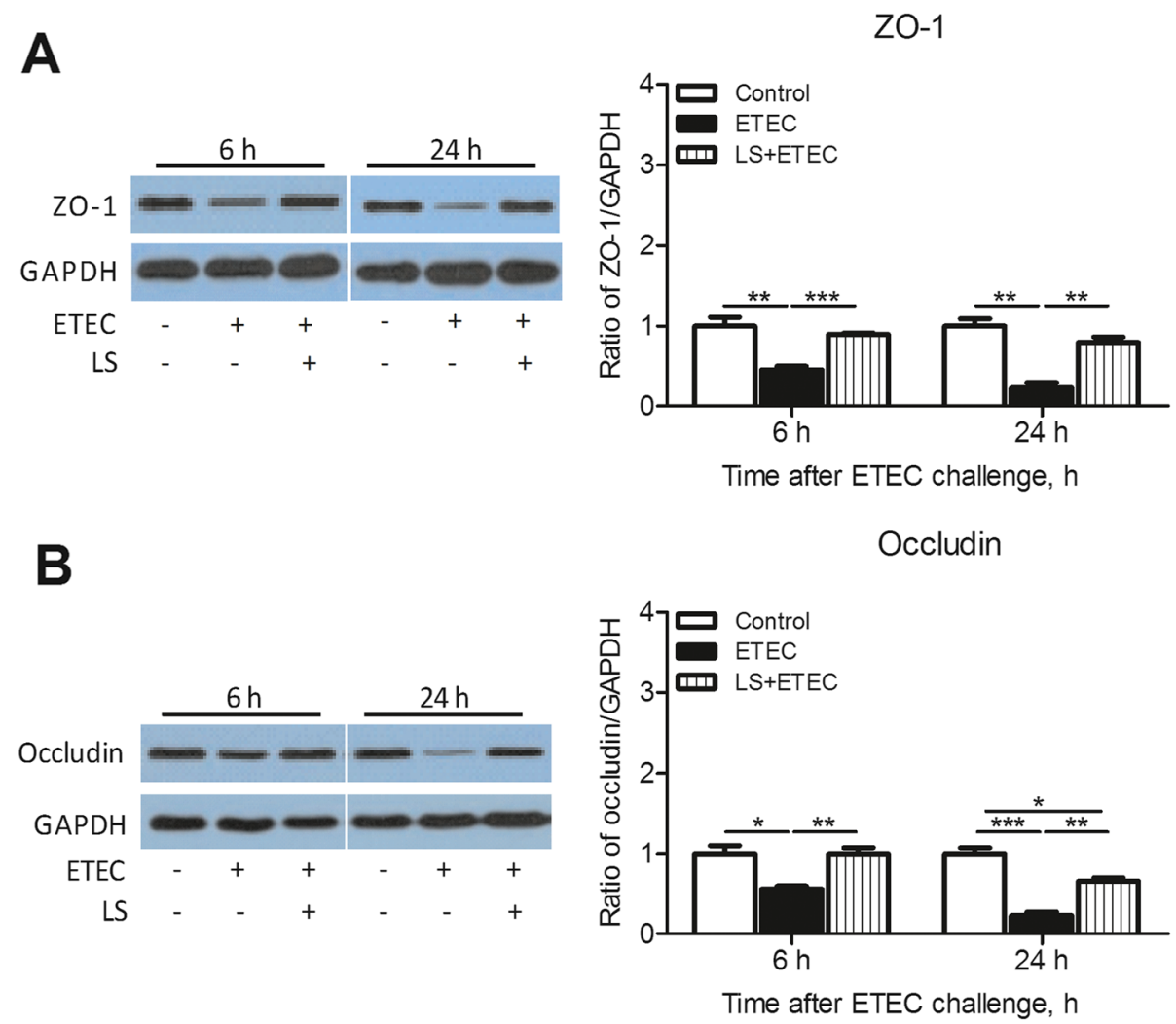

Fig. 5 Augmentation of tight junction proteins in IPEC-J2 cells treated with L. salivarius at 6 and $24 \mathrm{~h}$ after ETEC K88 challenge. (a) ZO-1 and (b) occludin were detected by Western blotting. GAPDH were used as the loading control. Data are presented as means \pm SEM of three independent experiments. ${ }^{*}, P<0.05 ;{ }^{*}, P<0.01 ;{ }^{* *}, P<0.001$

microorganisms for enterocyte binding. One previous study indicated that Lactobacillus might have a protective effect on the intestinal cells against the inflammatory status and mucosal injury triggered by ETEC K88 infection [22]. However, the basic molecular mechanism by which Lactobacillus might contribute to the regulation of intestinal epithelial cells still remains ambiguous and requires follow-up exploration.

The ability of the bacterial adhesion to host epithelial cells is regarded as one of the key steps for pathogenesis, which encourages some researches to focus on the adherent capability of ETEC K88 to IPEC-J2 cells with $L$. salivarius preincubation. One of our results demonstrated that $L$. salivarius administration made the adherent rate of ETEC K88 to IPEC-J2 cells drop 36\%, 33\%, $20 \%, 6 \%$ at $3,6,12$, and $24 \mathrm{~h}$ after infection, respectively (Fig. 1). The proteins anchored on the probiotic surface layer as well as some extracellular polysaccharides may prevent the formation of pathogenic biofilms by inhibiting adherence and blocking the binding sites of pathogenic bacteria to intestinal cell surface receptors. The adhesion of probiotic bacteria to epithelial cells also appear to be a contributing factor for optimizing bacterial colony balance in the pig intestine [23, 24]. Taken together, since $L$. salivarius could be able to effectively inhibit ETEC K88 adherence to porcine intestinal epithelial cells, this probiotic might have a defensive role in eliminating infectious process and improving the health of piglets.

Intestinal barrier is believed to be closely associated with functional tight junctions which are defined as dynamic complex locating between epithelial cells and function as a critical structure for paracellular permeability based on molecular charge and size [25]. In this sense, any alternation of this multi-functional and continuous assemble may contribute to the limitation of paracellular permeability and make entry or net flow of ions and solutes possible. Tight junctions comprise of transmembrane proteins (claudin and occludin) as well as various cytosolic proteins that are recruited to the apicolateral membrane, including zonula occludin protein 1 (ZO-1), ZO-2, ZO-3, cingulin, and 7H6 [26]. The integrity and regular permeability of tight junctions were negatively influenced in IPEC-J2 cells with invasion of ETEC K88 [27]. Plus, ETEC K88 infection could increase the permeability of tight junctions in early weaned piglets [28] and $L$. reuteri LR1 was proved to reduce ETEC K88-induced membrane barrier disruption by 

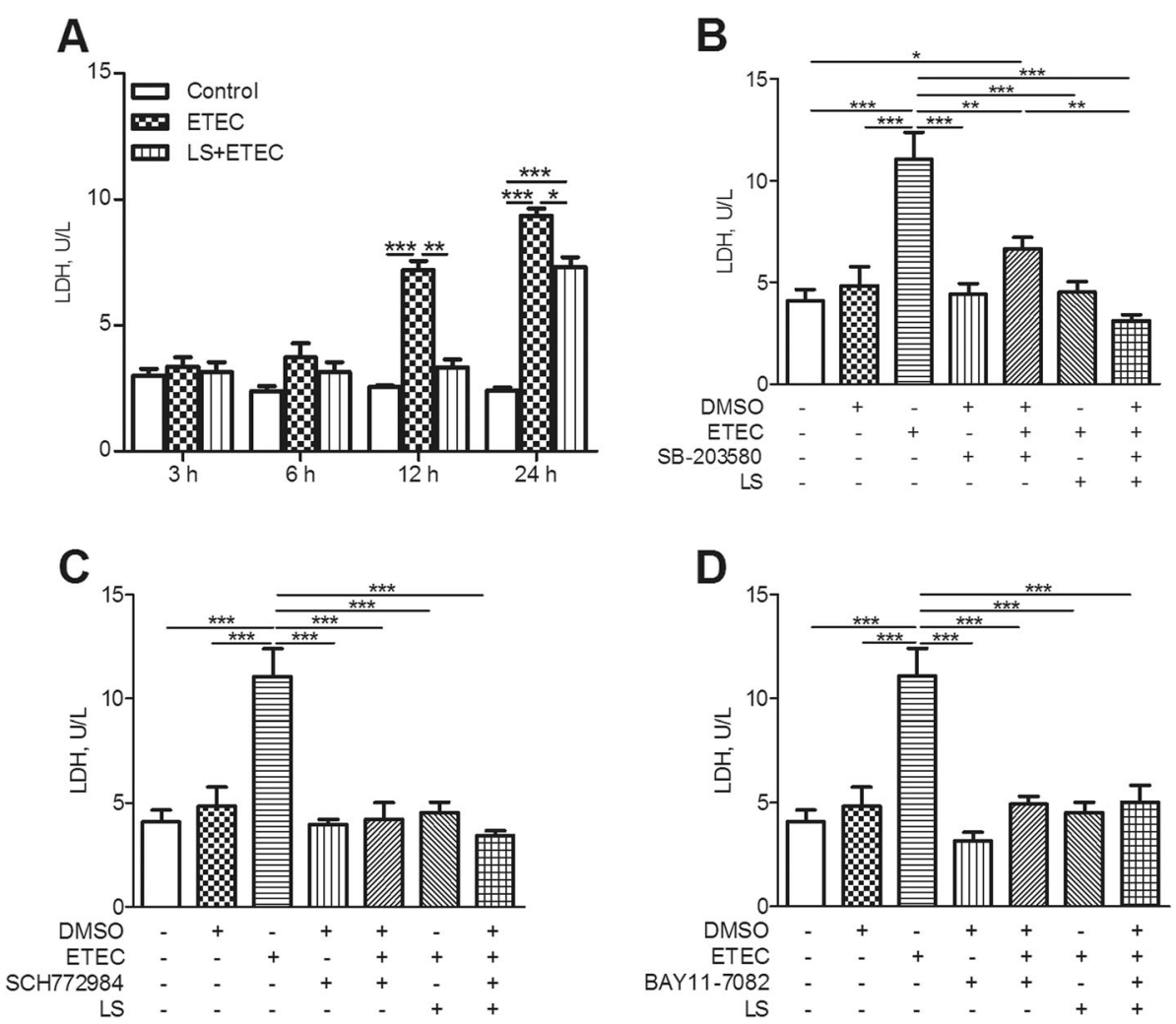

Fig. 6 Concentration of LDH in supernatants of IPEC-J2 cells. IPEC-J2 cells added with DMSO were regarded as the vehicle control and L. salivarius-involved IPEC-J2 cells were supplemented (a) without inhibitor, (b) with $10 \mu \mathrm{mol} / \mathrm{L}$ SB-203580 (p38 MAPK inhibitor), (c) with10 $\mu$ mol/L SCH772984 (ERK MAPK inhibitor) and (d) with $10 \mu \mathrm{mol} / \mathrm{L}$ BAY11-7082 (NF-KB inhibitor), respectively. Concentration of LDH was detected after ETEC K88 challenge. Data are presented as means \pm SEM of three independent experiments. ${ }^{*}, P<0.05 ;{ }^{* *}, P<0.01 ;{ }^{* *}, P<0.001$

maintaining proper position of $\mathrm{ZO}-1$ [29]. These supported evidences were consistent with our presented data that ETEC K88 challenge could decrease the expression of ZO-1 and occludin in IPEC-J2 cells, and these down-regulations could be partially reversed by the application of $L$. salivarius prior to bacterial infection (Fig. 5).

LDH is an oxidoreductase enzyme found in the cytoplasm and is widespread in tissues. Previous knowledge showed that both intracellular or extracellular stress resulted in an impaired mucosal barrier function and stimulated subsequent release of $\mathrm{LDH}$ from cells (as indicated by the amount of LDH in the culture medium) [30]. Thus, the amount of LDH detected in the culture medium is generally used as an indicator for determining damaged or dead cells. In our study, we found that ETEC K88 was able to induce inflammatory reaction in IPEC-J2 cells and highly augment LDH release from these cells. It's noteworthy to notice that the addition of $L$. salivarius to the culture medium markedly decreased LDH release from ETEC K88-induced cells (Fig. 6), which presented an involved role of L. salivarius in protecting the integrity of IPEC-J2 cell membrane.
The innate immune response is initiated by cell surface PRRs recognizing invading microorganisms and then down-streaming signals will be consequently activated to produce a variety of biological effects. TLRs and NLRs are two important PRRs and the roles of which in the immune system have been well established [31]. In the present study, ETEC K88 appeared to be a causing factor for the significant increase of TLR2 mRNA expression and the pretreatment of $L$. salivarius in ETEC K88-infected groups further elevated the mRNA expression of TLR2 within 3-12 h infection (Fig. 2a). L. paracasei was reported with an ability to inhibit proinflammatory cytokines by monocyte-macrophages via the induction of negative regulators for TLR2-dependent activation of NF- $\mathrm{kB}$ signaling pathways [32]. The decreased phosphorylation of $\mathrm{p} 65 \mathrm{NF}-\mathrm{kB}$ caused by $L$. salivarius might be related to the activation of TLR2. Thus, we hypothesize that activation of $L$. salivarius-mediated TLR2-dependent signaling pathways might induce the recruitment and activation of some inflammatory cells, serving as a protective and defensive strategy for host cells to resist ETEC K88-induced inflammation. Consistent with our results, the expression level of TLR2 in cells treated with either L. rhamnosus GR-1 [33] or $L$. 


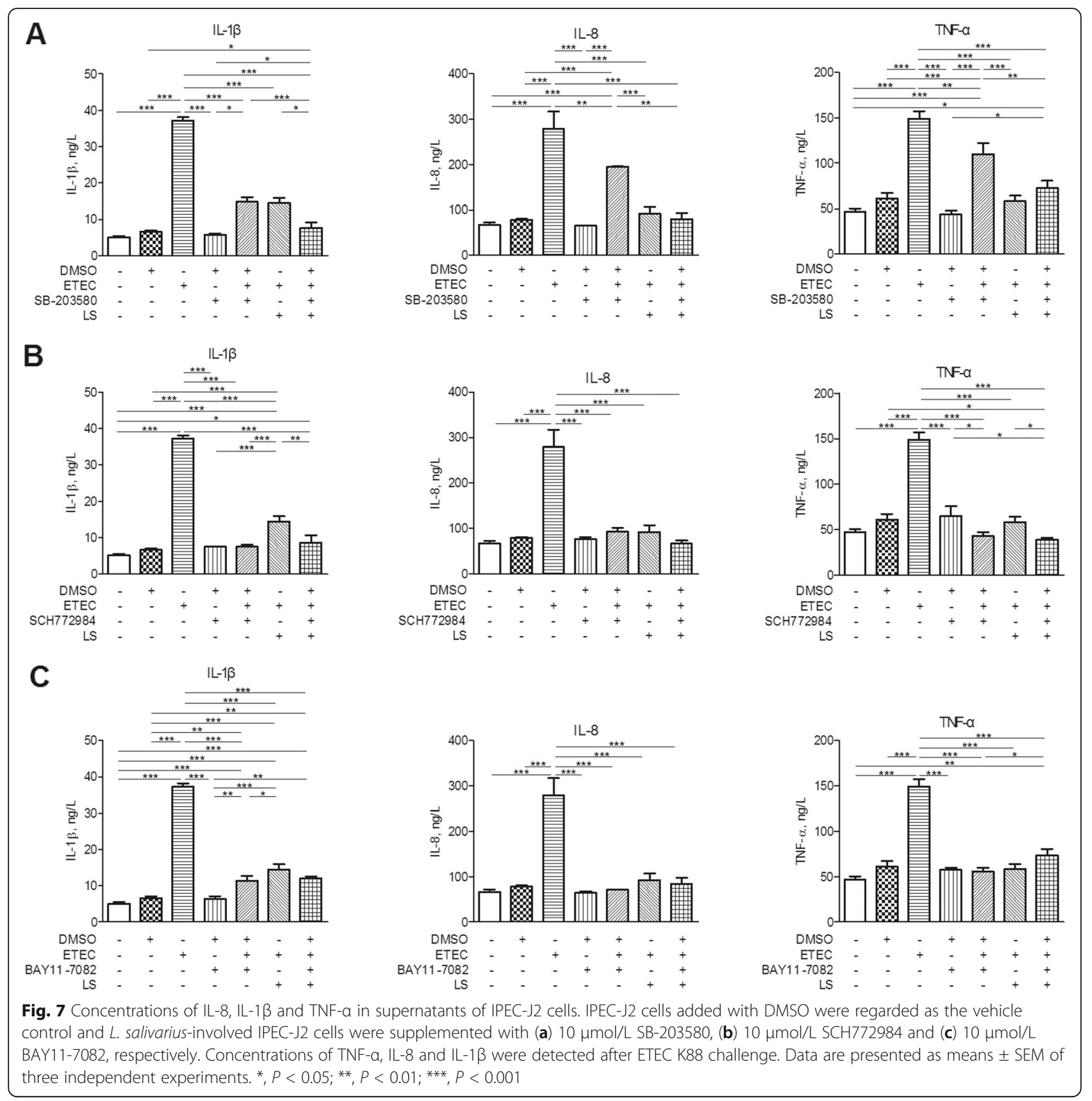

plantarum NDC 75017 [34] increased as well. However, an inhibitory impact of L. salivarius on TLR4 expression was shown in infectious IPEC-J2 cells, which was opposite to the change of TLR2 expression in the same group. Due to the fact that TLR4 is the main receptor for recognizing LPS, a vital component of Escherichia cell wall [35], it is no wonder that level of TLR4 in ETEC K88infected groups was relatively higher than that in the control groups in this study (Fig. 2b).

NLRs are cytoplasmic counterparts to TLRs and function in initiating the innate immune response with the appearance of pathogens [36]. NLRP3 and NLRP6 expression were both higher in groups with pathogenic involvement for 3-6 h, but this pattern started to decrease with an extended ETEC infectious time period for 12-24 h (Fig. 2 c, d). It is well appreciated that TLRs and NLRs are able to recruit adaptor protein MyD88 to activate a cascade of downstream signal transduction. For instance, MAPK and NF- $\mathrm{kB}$ are common targets for activation and mainly responsible for regulating related gene expression [15, 37]. A large number of investigators have confirmed the above statements and one study reported that the decreased expressions of TLRs and NLRs caused the attenuated phosphorylation of MAPK and 
NF- $\mathrm{kB}$, resulting in a decreased secretion of diverse cytokines [38]. Our data suggest that $L$. salivarius enabled the expression levels of TLR4, NLRP3 and NLRP6 to reduce in cells infected with ETEC K88. Previous data demonstrated the protective role of probiotics L.amylovorus and L. jensenii in down-regulating TLR4dependent NF- $\mathrm{kB}$ and MAPK activation as well as triggering negative regulators of TLRs, including Tollip, Bcl3, A20, MKP-1, and inhibitory IL-1R-related kinases [39, 40]. Their findings coupled with ours provide a possibility of probiotics to protect intestinal cells from ETEC K88 injury by alleviating inflammatory response. In addition, ERK1/2 and p38 are two distinct groups of MAPKs and involved in the inflammatory response. In this study, L. salivarius stimulation caused an inhibited phosphorylation of p38 MAPK in cells infected with ETEC for 1 or $3 \mathrm{~h}$. However, an enhancement of ERK phosphorylation was observed in the same group (Fig. 3a, b).

$\mathrm{NF}-\mathrm{kB}$, a transcription factor, is known as an important regulator for controlling the generation of multiple inflammation-related cytokines like TNF- $\alpha$. The production of TNF- $\alpha$ and the activation of NF- $\kappa B$ form a positive feedback loop in which the activation of NF-kB is followed by the production of TNF- $\alpha$ which further initiates signaling cascades and activates NF- $\mathrm{BB}$ [41]. Protein phosphorylation is central to the regulation of the signal transduction systems since it acts as a switch to turn protein activity on or off. Therefore, the phosphorylation of NF- $\kappa B$ subunit was measured in this research, and the result revealed the p65 subunit of NF- $\mathrm{kB}$ phosphorylation was significantly inhibited with $L$. salivarius treatment (Fig. 3c). Therefore, we speculate that L. salivarius might exert an anti-inflammatory effect in ETEC K88-induced IPEC-J2 cells at least in part via the NF- $\mathrm{KB}$ and MAPK-dependent signaling pathways.

Cytokine production is considered as an important indicator in response to ETEC K88. Even though some studies have demonstrated that LAB strains could trigger intestinal epithelial cells to produce pro-inflammatory cytokines [42, 43], we found that $L$. salivarius pretreatment prior to pathogenic exposure actually attenuated the release of cytokines IL-1 $\beta$, IL- 8 and TNF- $\alpha$ (Fig. 4). In line with our findings, $L$. plantarum could suppress proinflammatory cytokine production by inhibiting both NF- $\mathrm{kB}$ and p38 MAPK [44]. Other studies also demonstrated that the expression of pro-inflammatory cytokines like IL- 8 and IL- 6 would be down-regulated by $L$. reuteri [29]. These studies were confirmed by using the application of specific signaling inhibitors, which decreased cytokine levels in ETEC K88-induced cells (Fig. 7a, b, c). These results suggest that $L$. salivarius could regulate cytokines, probably slowing down cell damage and relatively weakening inflammation. In conclusion, our data suggest that $L$. salivarius might inhibit IL-1 $\beta$, IL- 8 , and TNF- $\alpha$ production due to the inhibition of NF- $\mathrm{kB}$ signaling pathway or the attenuated phosphorylation of p38 MAPK. It might also be possible for $L$. salivarius to enhance the cell integrity according to the up-regulated expression of $\mathrm{ZO}-1$ and occludin in tight junctions.

\section{Conclusions}

This study may provide useful information for the development of potential therapeutic strategies for the prevention or improvement of intestinal diseases in piglets using $L$. salivarius. At the same time, our data partly reveal the underlying mechanism of $L$. salivarius benefiting piglet health and provide a theoretical basis for the application of this probiotics in piglet diets.

\section{Abbreviations \\ CFU: Colony forming unit; ERK: Extracellular signal-regulated kinase; ETEC: Enterotoxigenic Escherichia coli; h: Hour; IL: Interleukin; IPEC- \\ J2: Intestinal porcine epithelial cell line; LB: Luria-Bertani; LDH: Lactate dehydrogenase; L. salivarius: Lactobacillus salivarius; MAPK: Mitogen-activated protein kinase; min: Minute; MRS: De Man, Rogosa and Sharpe; NF- \\ KB: Nuclear factor-KB; NLRP: NOD-like receptor pyrin domain-containing pro- tein; NLRs: NOD-like receptors; NOD: Nucleotide-binding oligomerization domain; PAMPs: Pathogen-associated molecular patterns; PBD-2: Porcine $\beta$ - defensin-2; PBS: Phosphate buffer saline; PCR: Polymerase chain reaction; PRRs: Pattern recognition receptors; PVDF: Polyvinylidene difluoride; RPMI: Roswell park memorial institute; TLR: Toll like receptor; TNF-a: Tumor necrosis factor-a; ZO-1: Zona occludens 1}

\section{Acknowledgements}

The authors would like to thank members of their laboratory for helpful and constructive advice.

\section{Authors' contributions}

$J \mathrm{Q}$ and $\mathrm{HL}$ designed the experiments. $\mathrm{JQ}, \mathrm{DL}$ and $\mathrm{HL}$ performed the experiments. JQ and $\mathrm{HL}$ analyzed the experimental data. $\mathrm{ZS}$ and $\mathrm{HL}$ wrote this paper. All authors read and approved the final manuscript.

\section{Funding}

This research was funded by the Tianjin Natural Science Foundation (18JCYBJC30000), the National Natural Science Foundation of China (31702147) and the Tianjin "131" Innovative Talents Team (20180338).

Availability of data and materials

All data generated or analyzed during this study are available from the corresponding author by request.

Ethics approval and consent to participate

Not applicable.

\section{Consent for publication}

Not applicable.

\section{Competing interests}

The authors declare that they have no competing interests.

\section{Author details}

${ }^{1}$ College of Life Sciences, Tianjin Key Laboratory of Animal and Plant Resistance, Tianjin Normal University, Tianjin 300387, People's Republic of China. ${ }^{2}$ Tianjin Key Laboratory of Agricultural Animal Breeding and Healthy Husbandry, College of Animal Science and Veterinary Medicine, Tianjin Agricultural University, 22 Jinjing Road, Tianjin 300384, People's Republic of China. 
Received: 3 March 2020 Accepted: 12 June 2020

\section{Published online: 03 August 2020}

\section{References}

1. Li HH, Jiang XR, Wang WJ, Qiao JY. Effects of Lactobacillus acidophilus and zinc oxide on the growth performance, jejunal morphology and immune function of weaned piglet following an Escherichia coli K88 challenge. Ital J Anim Sci. 2017;17:114-20.

2. Hou C, Zeng X, Yang F, Liu H, Qiao S. Study and use of the probiotic Lactobacillus reuteri in pigs: a review. J Anim Sci Biotechnol. 2015;6:14.

3. Qiao J, Li H, Wang Z, Wang W. Effects of Lactobacillus acidophilus dietary supplementation on the performance, intestinal barrier function, rectal microflora and serum immune function in weaned piglets challenged with Escherichia coli lipopolysaccharide. Antonie Van Leeuwenhoek. 2015;107: 883-91.

4. Tomosada Y, Villena J, Murata K, Chiba E, Shimazu T, Aso H, Iwabuchi N, et al. Immunoregulatory effect of bifidobacteria strains in porcine intestinal epithelial cells through modulation of ubiquitin-editing enzyme A20 expression. PLoS One. 2013:8:e59259.

5. Yang KM, Jiang ZY, Zheng CT, Wang L, Yang XF. Effect of Lactobacillus plantarum on diarrhea and intestinal barrier function of young piglets challenged with enterotoxigenic Escherichia coli K88. J Anim Sci. 2014;92: 1496-503.

6. Suda Y, Villena J, Takahashi Y, Hosoya S, Tomosada Y, Tsukida K, et al. Immunobiotic Lactobacillus jensenii as immune health promoting factor to improve growth performance and productivity in post-weaning pigs. BMC Immunol. 2014;15:24

7. Lahteinen T, Lindholm A, Rinttila T, Junnikkala S, Kant R, Pietila TE, et al. Effect of Lactobacillus brevis ATCC 8287 as a feeding supplement on the performance and immune function of piglets. Vet Immunol Immunopathol. 2014:158:14-25

8. Li H, Zhang L, Chen L, Zhu Q, Wang W, Qiao J. Lactobacillus acidophilus alleviates the inflammatory response to enterotoxigenic Escherichia coli K88 via inhibition of the NF-KB and p38 mitogen-activated protein kinase signaling pathways in piglets. BMC Microbiol. 2016;16:273.

9. Z Zhang J, Deng J, Li Y, Yang Q. The effect of Lactobacillus on the expression of porcine $\beta$-defensin-2 in the digestive tract of piglets. Livest Sci. 2011;138: 259-65

10. Zhang W, Zhu YH, Yang JC, Yang GY, Zhou D, Wang JF. A selected Lactobacillus rhamnosus strain promotes EGFR-independent Akt activation in an Enterotoxigenic Escherichia coli K88 infected IPEC-J2 cell model. PLoS One. 2015;10:e0125717.

11. Furuse M. Molecular basis of the Core structure of tight junctions. CSH Perspect Biol. 2010;2:a002907.

12. Miyauchi E, Morita H, Tanabe S. Lactobacillus rhamnosus alleviates intestinal barrier dysfunction in part by increasing expression of zonula occludens-1 and myosin light-chain kinase in vivo. J Dairy Sci. 2009;92:2400-8.

13. Corridoni D, Pastorelli L, Mattioli B, Locovei S, Ishikawa D, Arseneau KO, et al. Probiotic Bacteria regulate intestinal epithelial permeability in experimental ileitis by a TNF-dependent mechanism. PLoS One. 2012;7:e42067.

14. Miyauchi E, O'Callaghan J, Butto LF, Hurley G, Melgar S, Tanabe S, et al. Mechanism of protection of transepithelial barrier function by Lactobacillus salivarius: strain dependence and attenuation by bacteriocin production. Am J Physiol Gastrointest Liver Physiol. 2012;303:G1029-41.

15. Sun L, Pham TT, Cornell TT, McDonough KL, McHugh WM, Blatt NB, et al. Myeloid-specific gene deletion of protein phosphatase 2A magnifies MyD88- and TRIF-dependent inflammation following endotoxin challenge. J Immunol. 2017b;198:404-16.

16. Maisonneuve C, Bertholet S, Philpott DJ, De Gregorio E. Unleashing the potential of NOD- and Toll-like agonists as vaccine adjuvants. Proc Natl Acad Sci U S A. 2014;111:12294-9.

17. Yang F, Wang A, Zeng X, Hou C, Liu H, Qiao S. Lactobacillus reuteri 15007 modulates tight junction protein expression in IPEC-J2 cells with LPS stimulation and in newborn piglets under normal conditions. BMC Microbiol. 2015;15:32.

18. Mei HC, Liu YW, Chiang YC, Chao SH, Mei NW, Liu YW, et al. Immunomodulatory activity of Lactococcus lactis A17 from Taiwan fermented Cabbagein OVA-sensitized BALB/c mice. Evid Based Compl Alt. 2013;2013:287803.

19. Yang WS, Kim JJ, Lee MJ, Lee EK, Park SK. ADAM17-mediated Ectodomain shedding of toll-like receptor 4 as a negative feedback regulation in lipopolysaccharide-activated aortic endothelial cells. Cell Physiol Biochem. 2018:45:1851-62.

20. Bian J, Zhu S, Ma W, Li C, Ashraf MA. Analgesic effect and possible mechanism of SCH772984 intrathecal injection on rats with bone cancer pain. Saudi Pharm J. 2016;24:354-62.

21. Xia ZB, Meng FR, Fang YX, Wu X, Zhang CW, Liu Y, et al. Inhibition of NF-kB signaling pathway induces apoptosis and suppresses proliferation and angiogenesis of human fibroblast-like synovial cells in rheumatoid arthritis. Medicine (Baltimore). 2018;97:e10920.

22. Zhang W, Wang H, Liu J, Zhao Y, Gao K, Zhang J. Adhesive ability means inhibition activities for lactobacillus against pathogens and S-layer protein plays an important role in adhesion. Anaerobe. 2013;22:97-103.

23. Johnson-Henry KC, Hagen KE, Gordonpour M, Tompkins TA, Sherman PM. Surface-layer protein extracts from Lactobacillus helveticus inhibit enterohaemorrhagic Escherichia coli 0157:H7 adhesion to epithelial cells. Cell Microbiol. 2007;9:356-67.

24. Kim Y, Oh S, Kim SH. Released exopolysaccharide (r-EPS) produced from probiotic bacteria reduce biofilm formation of enterohemorrhagic Escherichia coli 0157:H7. Biochem Biophys Res Commun. 2009;379:324-9.

25. Yap AS, Mullin JM, Stevenson BR. Molecular analyses of tight junction physiology: insights and paradoxes. J Membr Biol. 1998;163:159-67.

26. Tsukita S, Furuse M, Itoh M. Structural and signaling molecules come together at tight junctions. Curr Opin Cell Biol. 1999:11:628-33.

27. Yu H, Ding X, Shang L, Zeng X, Liu H, Li N, et al. Protective ability of biogenic antimicrobial peptide microcin $\mathrm{J} 25$ against Enterotoxigenic Escherichia coli-induced intestinal epithelial dysfunction and inflammatory responses IPEC-J2 cells. Front Cell Infect Microbiol. 2018:8:242.

28. McLamb BL, Gibson AJ, Overman EL, Stahl C, Moeser AJ. Early weaning stress in pigs impairs innate mucosal immune responses to enterotoxigenic E coli challenge and exacerbates intestinal injury and clinical disease. PLoS One. 2013;8:e59838.

29. Wang Z, Wang L, Chen Z, Ma X, Yang X, Zhang J, et al. In vitro evaluation of swine-derived Lactobacillus reuteri: probiotic properties and effects on intestinal porcine epithelial cells challenged with Enterotoxigenic Escherichia coli K88. J Microbiol Biotechnol. 2016;26:1018-25.

30. Koh JY, Choi DW. Quantitative determination of glutamate mediated cortical neuronal injury in cell culture by lactate dehydrogenase efflux assay. J Neurosci Methods. 1987:20:83-90.

31. Mirpuri J, Sotnikov I, Myers L, Denning TL, Yarovinsky F, Parkos CA, et al. Lactobacillus rhamnosus (LGG) regulates IL-10 signaling in the developing murine colon through upregulation of the IL-10R2 receptor subunit. PLoS One. 2012;7:e51955

32. Sun KY, Xu DH, Xie C, Plummer S, Tang J, Yang XF, et al. Lactobacillus paracasei modulates LPS-induced inflammatory cytokine release by monocyte-macrophages via the up-regulation of negative regulators of NFkappaB signaling in a TLR2-dependent manner. Cytokine. 2017a;92:1-11.

33. Wu Q, Liu MC, Yang J, Wang JF, Zhu YH. Lactobacillus rhamnosus GR-1 ameliorates Escherichia coli-induced inflammation and cell damage via attenuation of ASC-independent NLRP3 inflammasome activation. Appl Environ Microbiol. 2015;82:1173-82

34. Jiang YJ, Li L, Sun HX, Shan Y, Liu Y, Li L, Man CX, et al. Induction of cytokines via NF-KB and p38 MAP kinase signalling pathways associated with the immunomodulation by Lactobacillus plantarum NDC 75017 in vitro and in vivo. J Funct Foods. 2016;20:215-25.

35. Janeway CA Jr, Medzhitov R. Innate immune recognition. Annu Rev Immunol. 2002;20:197-216.

36. Brubaker SW, Bonham KS, Zanoni I, Kagan JC. Innate immune pattern recognition: a cell biological perspective. Annu Rev Immunol. 2015:33:257-90.

37. Jin L, Batra S, Jeyaseelan S. Deletion of Nlrp3, augments survival during Polymicrobial Sepsis by decreasing autophagy and enhancing phagocytosis. J Immunol. 2017;198:1253-62.

38. Rathinam VA, Vanaja SK, Fitzgerald KA. Regulation of inflammasome signaling. Nat Immunol. 2012;13:333.

39. Shimazu T, Villena J, Tohno M, Fujie H, Hosoya S, Shimosato T, et al. Immunobiotic Lactobacillus jensenii elicits anti-inflammatory activity in porcine intestinal epithelial cells by modulating negative regulators of the toll-like receptor signaling pathway. Infect Immun. 2012;80:276-88.

40. Finamore A, Roselli M, Imbinto A, Seeboth J, Oswald IP, Mengheri E. Lactobacillus amylovorus inhibits the TLR4 inflammatory signaling triggered by enterotoxigenic Escherichia coli via modulation of the negative 
regulators and involvement of TLR2 in intestinal Caco-2 cells and pig explants. PLoS One. 2014;9:e94891.

41. Baeuerle PA, Henkel T. Function and activation of NF-KB in the immune system. Annu Rev Immunol. 1994;12:141-79.

42. Jiang Y, Lu X, Man C, Han L, Shan Y, Qu X, et al. Lactobacillus acidophilus induces cytokine and chemokine production via NF-KB and p38 mitogenactivated protein kinase signaling pathways in intestinal epithelial cells. Clin Vaccine Immunol. 2012;19:603-8.

43. Vizoso Pinto MG, Rodriguez Gomez M, Seifert S, Watzl B, Holzapfel WH, Franz CM. Lactobacilli stimulate the innate immune response and modulate the TLR expression of HT29 intestinal epithelial cells in vitro. Int J Food Microbiol. 2009;133:86-93.

44. Chon H, Choi B, Jeong G, Lee E, Lee S. Suppression of proinflammatory cytokine production by specific metabolites of Lactobacillus plantarum 10hk2 via inhibiting NF-KB and p38 MAPK expressions. Comp Immunol Microbiol Infect Dis. 2010;33:e41-9.

Ready to submit your research? Choose BMC and benefit from:

- fast, convenient online submission

- thorough peer review by experienced researchers in your field

- rapid publication on acceptance

- support for research data, including large and complex data types

- gold Open Access which fosters wider collaboration and increased citations

- maximum visibility for your research: over $100 \mathrm{M}$ website views per year

At $\mathrm{BMC}$, research is always in progress.

Learn more biomedcentral.com/submissions 